\title{
Anti-obesity effects of Actinidia polygama extract in mice with high-fat diet-induced obesity
}

\author{
YOON-YOUNG SUNG ${ }^{1}$, TAESOOK YOON ${ }^{1}$, WON-KYUNG YANG ${ }^{1}$, \\ BYEONG-CHEOL MOON $^{1}$ and HO KYOUNG KIM ${ }^{1,2}$ \\ ${ }^{1}$ Basic Herbal Medicine Research Group; ${ }^{2}$ Herbal Material Management Team, \\ Korea Institute of Oriental Medicine, Daejeon 305-811, Republic of Korea
}

Received September 10, 2012; Accepted December 5, 2012

DOI: $10.3892 / \mathrm{mmr} .2012 .1239$

\begin{abstract}
Actinidia polygama has been used as a herbal folk medicine for treating pain, gout, rheumatoid arthritis and inflammation. In the present study, the anti-obesity properties of Actinidia polygama extract (APE) were investigated in mice with high-fat diet-induced obesity. APE treatment of high-fat diet (HFD)-fed obese mice significantly reduced body weight, adipose tissue mass and serum triglyceride and leptin levels relative to the HFD-fed mice. Food intake did not differ between the HFD and HFD+APE groups, although the food efficiency ratio (FER) was significantly decreased in the HFD+APE group compared with the HFD group. Histological examination showed that the sizes of the adipocytes were significantly smaller in the HFD+APE group compared with the HFD group. Serum levels of aspartate transaminase were significantly decreased in the HFD+APE mice compared with the HFD-fed mice, but serum levels of alanine transaminase (ALT), blood urea nitrogen and creatinine were not significantly changed in the HFD+APE mice compared with the levels in the normal diet (ND)-fed and HFD-fed mice. These results suggest that APE may be useful for treating metabolic diseases, including obesity and hyperlipidemia, without toxic side-effects.
\end{abstract}

\section{Introduction}

Obesity, which is caused by an imbalance between energy intake and expenditure, is associated with various metabolic

Correspondence to: Dr Ho Kyoung Kim, Basic Herbal Medicine Research Group, Korea Institute of Oriental Medicine, 483 Exporo, Yuseong-gu, Daejeon 305-811, Republic of Korea

E-mail: hkkim@kiom.re.kr

Abbreviations: ALT, alanine transaminase; APE, Actinidia polygama extract; AST, aspartate transaminase; BPT, Bangpoongtongsungsan; FER, food efficiency ratio; HDL, high-density lipoprotein; HFD, high-fat diet; LDL, low-density lipoprotein; ND, normal diet; WAT, white adipose tissue

Key words: Actinidia polygama, body weight, fat, leptin, triglyceride diseases, including hypertension, dyslipidemia, diabetes mellitus, coronary heart disease, congestive heart failure, stroke, osteoarthritis, sleep apnea and certain types of cancer (e.g., colon, breast, endometrial and gall bladder) (1).

Numerous pharmacological approaches for the prevention and treatment of obesity have been suggested. Obesity therapies include the suppression of nutrient absorption and the administration of drugs that control lipid utilization (2). Clinically available anti-obesity agents include orlistat, which is a lipase inhibitor, and sibutramine, which is a centrally acting inhibitor of serotonin and norepinephrine uptake $(3,4)$. However, these agents have been reported to cause undesirable side-effects, including constipation, insomnia, vomiting, headaches, stomachaches and heart attacks (5). Therefore, due to the limited usage of these pharmacological agents, there is a demand for alternative therapies, including the use of herbal products, that have minimal side-effects (6). Bangpoongtongsungsan (BPT), a traditional herbal medicine composed of 18 crude drugs, has been reported to be an effective treatment for obesity, insulin resistance and hypertension (7). In particular, BPT decreases the weight of white adipose tissues (WATs) and the size of adipocytes without adverse effects in high-fat diet (HFD)-fed mice (8).

Actinidia polygama Miquel (actinidiaceae) fructus has been used as a herbal folk medicine for treating pain, gout, rheumatoid arthritis and inflammation (9). It has been reported that A. polygama extract (APE) exhibits inhibitory activity against rat paw edema induced by carrageenans and that it inhibits lipopolysaccharide-induced nitric oxide (NO) production in RAW 264.7 macrophages (10). Additionally, APE demonstrates anti-inflammatory and anti-asthmatic effects in a murine model of asthma (11). However, the anti-obesity effects of A. polygama have not previously been studied.

In the present study, the activities of a $70 \%$ ethanol extract of A. polygama in changes in body weight, fat accumulation and serum lipid levels were elucidated in the mouse model of HFD-induced obesity.

\section{Materials and methods}

Preparation of APE. A. polygama fructus was purchased as a dried herb from Hanherb Co. (Seoul, Korea) and authenticated based on its microscopic and macroscopic characteristics by 
Table I. Effects of APE on body weight and food intake in mice with HFD-induced obesity.

\begin{tabular}{lccccc}
\hline Factor & ND & HFD & HFD+APE & HFD+orlistat & HFD+BPT \\
\hline Initial body weight (g) & $19.64 \pm 0.21$ & $19.64 \pm 0.19$ & $19.64 \pm 0.18$ & $19.64 \pm 0.20$ & $19.64 \pm 0.20$ \\
Final body weight (g) & $24.18 \pm 0.26$ & $29.17 \pm 0.48^{\mathrm{c}}$ & $26.97 \pm 0.71^{\mathrm{d}}$ & $28.07 \pm 0.17$ & $28.11 \pm 0.98$ \\
Body weight gain $^{\mathrm{a}}$ (g/7 weeks) & $4.54 \pm 0.16$ & $9.63 \pm 0.32^{\mathrm{c}}$ & $7.34 \pm 0.60^{\mathrm{e}}$ & $8.37 \pm 0.25^{\mathrm{d}}$ & $8.49 \pm 0.81$ \\
Food intake (g/day) $_{\text {FER }^{\mathrm{b}}}^{4.23 \pm 0.13}$ & $2.33 \pm 0.02^{\mathrm{c}}$ & $2.58 \pm 0.27$ & $2.35 \pm 0.03$ & $2.29 \pm 0.10$ \\
& $0.022 \pm 0.001$ & $0.084 \pm 0.002^{\mathrm{c}}$ & $0.060 \pm 0.006^{\mathrm{e}}$ & $0.074 \pm 0.002^{\mathrm{d}}$ & $0.076 \pm 0.006^{\mathrm{d}}$ \\
\hline
\end{tabular}

${ }^{\mathrm{a} B o d y}$ weight gain = final body weight - initial body weight. ${ }^{\mathrm{b}} \mathrm{FER}($ food efficiency ratio) $=$ body weight gain for experimental period $(\mathrm{g} / \mathrm{day}) /$ food intake for experimental period (g/day). Values are expressed as mean \pm standard error $(\mathrm{n}=7) .{ }^{\mathrm{c}} \mathrm{P}<0.001$ vs. $\mathrm{ND}$ group. ${ }^{\mathrm{d}} \mathrm{P}<0.05$, ${ }^{\mathrm{e}} \mathrm{P}<0.01 \mathrm{vs}$. HFD group. ND, normal diet; HFD, high-fat diet; APE, Actinidia polygama extract; BPT, Bangpoongtongsungsan.

the Classification and Identification Committee of the Korea Institute of Oriental Medicine (KIOM). The committee was composed of nine experts in the fields of plant taxonomy, botany, pharmacognosy and herbology. A voucher specimen (no. JA-85) was deposited at the herbarium of the Department of Herbal Resources Research in KIOM. Extracts from dried fructus of A. polygama (300 g) were obtained twice with $70 \%$ ethanol (with 2-h reflux) and the extract was then concentrated under reduced pressure. The decoction was filtered, lyophilized and serially stored at $4^{\circ} \mathrm{C}$. The yield of the dried extract from the starting crude materials was $\sim 14.78 \%$ (w/w).

Animals and diets. Male 4-week-old C57BL/6J mice were purchased from Daehan Biolink Co. (Eumsung, Korea) and acclimated for 1 week prior to the experiments. The mice were housed in an air-conditioned animal room with a 12 -h light/12-h darkness cycle at a temperature of $22 \pm 1^{\circ} \mathrm{C}$ and humidity of $50 \pm 10 \%$. The mice were provided with a laboratory diet and water ad libitum. All experimental protocols involving the use of animals were conducted in accordance with the National Institutes of Health (NIH) guidelines and approved by the Committee on Animal Care for our institute.

To induce obesity, the mice were fed a HFD (Rodent diet D12492, Research Diet, New Brunswick, NJ, USA) consisting of $60 \%$ energy as fat, $20 \%$ as protein and $20 \%$ as carbohydrates, in accordance with previously published studies (12). The control mice were fed a commercial standard chow diet (Orient Bio, Inc., Seongnam, Korea) consisting of 14\% energy as fat, $21 \%$ as protein and $65 \%$ as carbohydrates. Orlistat and $\mathrm{BPT}$ were used as positive controls. The mice were randomly divided into five groups $(n=7)$ and fed a normal diet (ND), a HFD, a HFD plus APE (HFD+APE), a HFD plus orlistat (HFD+orlistat) or a HFD plus BPT (HFD+BPT) for 7 weeks. APE or BPT was dissolved in normal saline and orally administered to the mice at a dose of $400 \mathrm{mg} / \mathrm{kg} / \mathrm{day}$ for 7 weeks. Orlistat was administered to the mice at a dose of $15.9 \mathrm{mg} / \mathrm{kg} /$ day. By contrast, the vehicle (normal saline) was orally administered to the mice in the ND-fed and HFD-fed control groups. Body weight and food intake were measured twice a week.

Biochemical analysis of blood. At the end of the experimental period, the mice were fasted prior to being sacrificed. The mice were anesthetized with ether and blood samples were obtained from the inferior vena cava of each mouse. The blood samples were centrifuged at $2,000 \mathrm{x}$ g for $15 \mathrm{~min}$ at $4^{\circ} \mathrm{C}$, then the serum was collected and stored at $-70^{\circ} \mathrm{C}$ prior to analysis.

The serum levels of the triglycerides, total cholesterol, low-density lipoprotein (LDL)-cholesterol, high-density lipoprotein (HDL)-cholesterol, aspartate transaminase (AST), alanine transaminase (ALT), blood urea nitrogen and creatinine were analyzed with an automated serum analyzer (Hitachi 7080, Hitachi Co., Tokyo, Japan). The concentrations of serum leptin and adiponectin were measured with mouse leptin and adiponectin ELISA kits (R\&D Systems, Minneapolis, MN, USA), respectively, according to the manufacturer's instructions. Absorbance values were measured using a microplate spectrophotometer (Bio-Rad, Hercules, CA, USA).

Adipose tissue weight and histological analysis. Subsequent to blood collection, the WATs (subcutaneous, epididymal and retroperitoneal) were removed from the mice and immediately weighed.

To stain the adipocytes, the adipose tissues were fixed in $10 \%$ neutral formalin solution for 1 day and embedded in paraffin. All tissues were cut to a thickness of $6 \mu \mathrm{m}$ and stained with hematoxylin and eosin. To quantify the adipocyte sizes, the stained sections were analyzed using light microscopy (Olympus BX51, Olympus Optical Co., Tokyo, Japan) and an image analysis program (Image pro plus 5.0, Media Cybernetics, Silver Spring, MD, USA).

Statistical analysis. Differences between the groups were examined using an unpaired Student's t-test and analysis of variance (ANOVA) followed by Duncan's multiple range test. All data are presented as mean \pm standard error $(\mathrm{SE}) . \mathrm{P}<0.05$ was considered to indicate a statistically significant difference.

\section{Results}

Effects of APE on body weight and food intake. The body weights and food intake of the mice in the five treatment groups are listed in Table I. Subsequent to the 7-week treatment, the increase in body weight of the HFD group was significantly greater than that of the ND group $(\mathrm{P}<0.001)$. However, the increase in body weight of the HFD+APE group was significantly less than that of the HFD group $(\mathrm{P}<0.01)$. During the feeding period, food intake was higher in the ND group than 
Table II. Effects of APE on organ weights in mice with HFD-induced obesity ( $\mathrm{g} / 100 \mathrm{~g}$ body weight).

\begin{tabular}{|c|c|c|c|c|c|}
\hline \multirow[b]{2}{*}{ Type of tissue } & \multicolumn{5}{|c|}{ WAT weights } \\
\hline & ND & HFD & HFD+APE & HFD+orlistat & $\mathrm{HFD}+\mathrm{BPT}$ \\
\hline Subcutaneous WAT & $0.94 \pm 0.04$ & $2.85 \pm 0.54^{\mathrm{a}}$ & $2.17 \pm 0.14$ & $2.43 \pm 0.15$ & $2.92 \pm 0.42$ \\
\hline Epididymal WAT & $1.13 \pm 0.14$ & $5.29 \pm 0.58^{b}$ & $3.44 \pm 0.35^{\mathrm{c}}$ & $4.19 \pm 0.15$ & $4.78 \pm 0.81$ \\
\hline Retroperitoneal WAT & $0.36 \pm 0.11$ & $2.22 \pm 0.39^{b}$ & $1.19 \pm 0.11^{\mathrm{c}}$ & $1.56 \pm 0.13$ & $1.68 \pm 0.30$ \\
\hline
\end{tabular}

Values are expressed as mean \pm standard error $(\mathrm{n}=5)$. ${ }^{\mathrm{a}} \mathrm{P}<0.01,{ }^{\mathrm{b}} \mathrm{P}<0.001$ vs. ND group and ${ }^{\mathrm{c}} \mathrm{P}<0.05$ vs. HFD group. ND, normal diet; HFD, high-fat diet; APE, Actinidia polygama extract; BPT, Bangpoongtongsungsan; WAT, white adipose tissue.

Table III. Effects of APE on biochemical parameters in serum of mice with HFD-induced obesity.

\begin{tabular}{|c|c|c|c|c|c|}
\hline Parameter & ND & HFD & $\mathrm{HFD}+\mathrm{APE}$ & HFD+orlistat & HFD+BPT \\
\hline Total cholesterol (mg/dl) & $121 \pm 2.6$ & $161 \pm 2.7^{b}$ & $157 \pm 12.1$ & $147 \pm 4.1^{\mathrm{c}}$ & $151 \pm 7.8$ \\
\hline Triglyceride (mg/dl) & $68 \pm 23.3$ & $83 \pm 4.5^{\mathrm{a}}$ & $57 \pm 7.7^{c}$ & $76 \pm 9.8$ & $51 \pm 4.9^{\mathrm{d}}$ \\
\hline LDL-cholesterol (mg/dl) & $4.2 \pm 0.5$ & $4.8 \pm 0.3$ & $6.6 \pm 1.3$ & $4.7 \pm 0.2$ & $5.8 \pm 0.6$ \\
\hline HDL-cholesterol (mg/dl) & $64.4 \pm 1.9$ & $66.6 \pm 1.0$ & $65.3 \pm 5.0$ & $68.2 \pm 2.3$ & $65.9 \pm 3.0$ \\
\hline Adiponectin $(\mu \mathrm{g} / \mathrm{ml})$ & $12.27 \pm 0.54$ & $13.43 \pm 0.44$ & $13.20 \pm 0.19$ & $11.51 \pm 0.9$ & $12.85 \pm 0.5$ \\
\hline Leptin $(\mathrm{ng} / \mathrm{ml})$ & $4.09 \pm 0.69$ & $19.40 \pm 3.20^{\mathrm{b}}$ & $9.44 \pm 0.97^{\mathrm{c}}$ & $9.17 \pm 0.9^{c}$ & $10.87 \pm 2.8$ \\
\hline
\end{tabular}

Values are expressed as mean \pm standard error $(\mathrm{n}=7)$. ${ }^{\mathrm{a}} \mathrm{P}<0.01$ and ${ }^{\mathrm{b}} \mathrm{P}<0.001$ vs. ND group. ${ }^{\mathrm{c}} \mathrm{P}<0.05$ and ${ }^{\mathrm{d}} \mathrm{P}<0.01$ vs. HFD group. ND, normal diet; HFD, high-fat diet; APE, Actinidia polygama extract; BPT, Bangpoongtongsungsan; LDL, low-density lipoprotein; HDL, high-density lipoprotein.
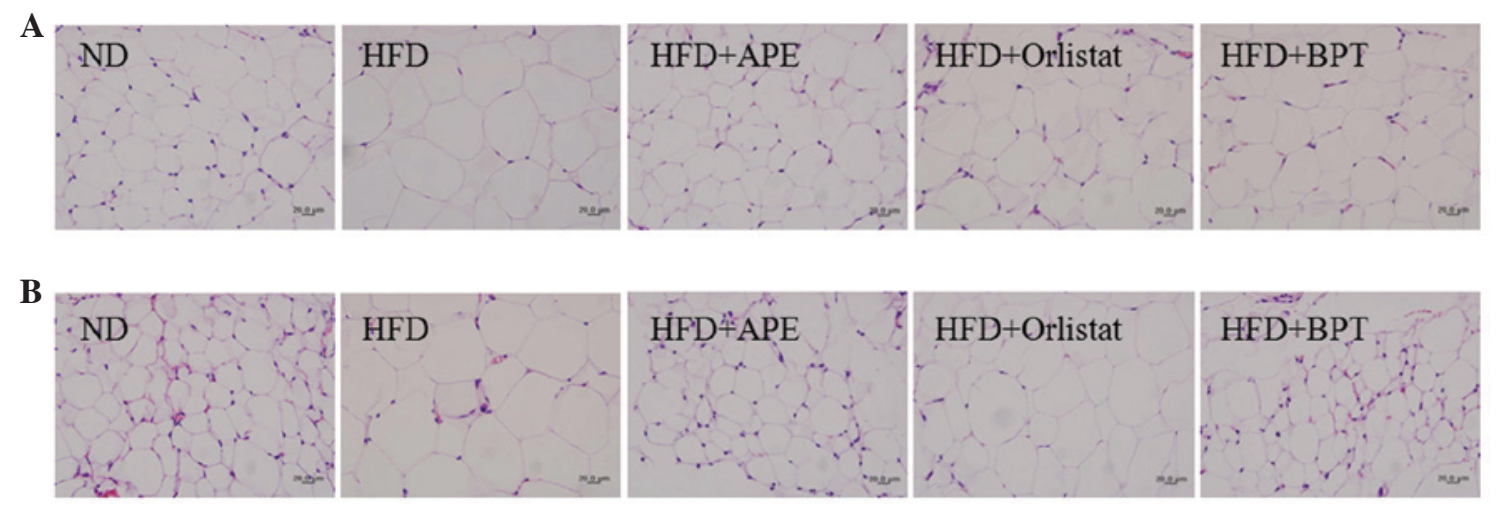

Figure 1. Histology of white adipose tissue (WAT) in mice with HFD-induced obesity. APE was orally administered to mice at a dose of $400 \mathrm{mg} / \mathrm{kg} / \mathrm{day}$ for 7 weeks. Representative images of hematoxylin and eosin-stained sections (magnification, x400) of (A) epididymal and (B) retroperitoneal WAT. ND, normal diet; HFD, high-fat diet; APE, Actinidia polygama extract; BPT, Bangpoongtongsungsan.

in the HFD group, but did not differ significantly between the HFD and HFD+APE groups. The food efficiency ratio (FER) was higher in the HFD group than in the ND group, whereas the FER was significantly lower in the HFD+APE mice than in the HFD group $(\mathrm{P}<0.01)$.

Effects of APE on WAT weights. The WAT weights are listed in Table II. The weights of various WATs, including subcutaneous, epididymal and retroperitoneal adipose tissues, were greater in the HFD-fed mice than in the ND-fed mice, while the weights of epididymal and retroperitoneal adipose tissues were significantly lower in the HFD+APE group $(\mathrm{P}<0.05)$.
Histological analysis of WAT. Adipose tissues were further examined histologically (Fig. 1). The sizes of the retroperitoneal and epididymal adipocytes were larger in the HFD group than in the ND group. APE treatment decreased the sizes of the adipocytes in the obese mice relative to the adipocytes in the HFD-fed mice.

Effects of APE on serum biochemical levels. The serum biochemical parameters are listed in Table III. The serum triglyceride, total cholesterol and leptin levels were significantly increased in the HFD group compared with the ND group. APE treatment of the HFD-fed mice significantly 
Table IV. Effects of APE on toxicological parameters in serum of mice with HFD-induced obesity.

\begin{tabular}{llllll}
\hline Parameter & \multicolumn{1}{c}{ ND } & HFD & HFD+APE & HFD+orlistat & HFD+BPT \\
\hline AST (U/l) & $90.6 \pm 13.8$ & $124.8 \pm 22.4$ & $62.3 \pm 10.0^{\mathrm{b}}$ & $80.0 \pm 11.9$ & $78.5 \pm 23.6$ \\
ALT (U/l) & $46.4 \pm 2.5$ & $35.0 \pm 9.9$ & $20.9 \pm 1.7$ & $25.4 \pm 1.9$ & $24.7 \pm 0.5$ \\
Blood urea nitrogen (mg/dl) & $31.6 \pm 1.1$ & $21.4 \pm 1.3^{\mathrm{c}}$ & $20.1 \pm 2.0$ & $20.1 \pm 1.1$ & $21.0 \pm 1.2$ \\
Creatinine (mg/dl) & $0.45 \pm 0.02$ & $0.41 \pm 0.22$ & $0.38 \pm 0.03$ & $0.43 \pm 0.03$ & $0.48 \pm 0.02$
\end{tabular}

Values are expressed as mean \pm standard error $(\mathrm{n}=7)$. ${ }^{\mathrm{P}}<0.001$ vs. ND group. ${ }^{\mathrm{b}} \mathrm{P}<0.05$ vs. HFD group. ND, normal diet; HFD, high-fat diet; APE, Actinidia polygama extract; BPT, Bangpoongtongsungsan; AST, aspartate transaminase; ALT, alanine transaminase.

reduced the triglyceride and leptin levels $(\mathrm{P}<0.05)$. However, the total cholesterol levels did not differ between the HFD and HFD+APE groups. The HDL-cholesterol, LDL-cholesterol and adiponectin levels did not change among the five groups.

Assessment of potential toxicological effects. The effects of APE on the toxicological parameters are listed in Table IV. To evaluate the potential toxic effects of APE ingestion, serum toxicological markers, which indicate liver and kidney injury, were measured at the end of the experimental period. The AST levels were significantly lower in the HFD+APE mice than in the HFD mice $(\mathrm{P}<0.05)$. The serum concentrations of ALT, blood nitrogen urea and creatinine were not significantly changed in the HFD+APE group compared with the HFD group. AST, ALT, blood nitrogen urea and creatinine levels were within previously published normal ranges for all groups $(13,14)$. Additionally, HFD+APE mice did not exhibit significant changes in the liver and spleen weights (data not shown). These data indicate that administration of $400 \mathrm{mg} / \mathrm{kg} /$ day APE for 7 weeks induced no detectable adverse toxic effects in the mice.

\section{Discussion}

The results of the present study demonstrated that APE treatment inhibited body weight gain, visceral (epididymal and retroperitoneal) adipose tissue mass, adipocyte size and serum triglyceride levels in mice with HFD-induced obesity. APE treatment in the HFD-fed mice did not change the food intake levels but decreased the FER, implying that APE-treated, HFD-fed mice were less efficient at converting nutrients into their own body mass. These results show that APE has potent hypolipidemic and anti-obesity effects. In addition, the 7-week APE treatment induced no detectable adverse toxic effects in the mice. These data suggest that the decreased fat mass observed in APE-treated mice was not due to the toxicity of APE but rather due to a direct pharmacological action on the adipose tissues.

APE treatment in HFD-fed mice reduced the serum triglyceride and leptin concentrations with decreased fat masses. Furthermore, APE treatment in 3T3-L1 preadipocytes inhibited adipocyte differentiation and lipid (triglyceride) accumulation (data not shown). Moreover, histological examination revealed a decrease in the adipocyte size in the HFD+APE group compared with that of the HFD group. Adipose tissue is known for its capacity to store excess dietary energy in the form of triglycerides within the lipid droplets of adipocytes (15).
Obesity is characterized by increased adipose tissue mass that results from an increased fat cell number (hyperplasia) and an increased fat cell size (hypertrophy) (16). Specifically, visceral obesity due to adipocyte hypertrophy is more closely associated with various metabolic and circulatory diseases (17).

Visceral fat accumulation induces the production of a variety of proteins known as adipokines, including visfatin, tumor necrosis factor- $\alpha$, interleukin-6, leptin and adiponectin (18). The secretion levels of leptin, a key fat-derived regulator of food intake and energy expenditure, are positively correlated with adiposity and body weight changes in humans and rodents (19). In the present study, the serum leptin levels were decreased in the HFD+APE group compared with the levels in the HFD group. Adiponectin is known to contribute to insulin sensitivity and fatty acid oxidation and adiponectin levels are decreased in obesity, type 2 diabetes and atherosclerosis $(20,21)$. However, the levels of adiponectin in the present study were not significantly changed in the HFD+APE mice compared with levels in the HFD-fed mice. This result suggests that the decreased serum leptin levels from the APE treatment in the HFD-fed mice may be attributable to a decreased lipid accumulation in the adipose tissues.

In conclusion, the APE treatment reduced body weight gain, adipose tissue accumulation, adipocyte size and serum triglyceride and leptin levels without toxic effects in mice with HFD-induced obesity. The potent effect of APE on adipocyte composition and lipidemia without apparent toxic side-effects makes APE a promising candidate in the development of anti-obesity pharmacotherapy.

\section{Acknowledgements}

This study was supported by the Discovery of Herbal Medicine for the 'Prevention of Prehypertension Project' (K12202) and the 'Construction of the Basis for Practical Application of Herbal Resources' (K12020) funded by the Ministry of Education, Science and Technology (MEST) of Korea to the Korea Institute of Oriental Medicine (KIOM).

\section{References}

1. Devlin MJ, Yanovski SZ and Wilson GT: Obesity: what mental health professionals need to know. Am J Psychiatry 157: 854-866, 2000.

2. Lee YS, Cha BY, Saito K, Choi SS, Wang XX, Choi BK, Yonezawa T, Teruya T, Nagai K and Woo JT: Effects of a Citrus depressa Hayata (shiikuwavsa) extract on obesity in high-fat diet-induced obese mice. Phytomedicine 18: 648-654, 2011. 
3. Hofbauer KG, Nicholson JR and Boss O: The obesity epidemic: current and future pharmacological treatments. Annu Rev Pharmacol Toxicol 47: 565-592, 2007.

4. Sharma B and Henderson DC: Sibutramine: current status as an anti-obesity drug and its future perspectives. Expert Opin Pharmacother 9: 2161-2173, 2008.

5. Bray GA: Drug treatment of obesity. Rev Endocr Metab Disord 2: 403-418, 2001.

6. Song MY, Lv N, Kim EK, Kwon KS, Yoo YB, Kim JH, Lee SW, Song JH, Lee JH, Lee SK, Shin BC, Ryu DG, Park BH and Kwon KB: Antiobesity activity of aqueous extracts of Rhizoma Dioscoreae Tokoronis on high-fat diet-induced obesity in mice. J Med Food 12: 304-309, 2009.

7. Shimada T, Kudo T, Akase T and Aburada M: Preventive effects of Bofutsushosan on obesity and various metabolic disorders Biol Pharm Bull 31: 1362-1367, 2008

8. Akagiri S, Naito Y, Ichikawa H, Mizushima K, Takagi T, Handa O, Kokura S and Yoshikawa T: Bofutsushosan, an oriental herbal medicine, attenuates the weight gain of white adipose tissue and the increased size of adipocytes associated with the increase in their expression of uncoupling protein 1 in high-fat diet-fed male KK/Ta mice. J Clin Biochem Nutr 42: 158-166, 2008.

9. Bang MH, Chae IG, Lee EJ, Baek NI, Baek YS, Lee DY, Lee IS, Lee SP and Yang SA: Inhibitory effects of actinidiamide from Actinidia polygama on allergy and inflammation. Biosci Biotechnol Biochem 76: 289-293, 2012.

10. Kim YK, Kang HJ, Lee KT, Choi JG and Chung SH: Antiinflammation activity of Actinidia polygama. Arch Pharm Res 26: 1061-1066, 2003.

11. Lee YC, Kim SH, Seo YB, Roh SS and Lee JC: Inhibitory effects of Actinidia polygama extract and cyclosporine A on OVA-induced eosinophilia and bronchial hyperresponsiveness in a murine model of asthma. Int Immunopharmacol 6: 703-713, 2006.

12. Choi H, Eo H, Park K, Jin M, Park EJ, Kim SH, Park JE and Kim S: A water-soluble extract from Cucurbita moschata shows anti-obesity effects by controlling lipid metabolism in a high fat diet-induced obesity mouse model. Biochem Biophys Res Commun 359: 419-425, 2007.
13. Han SS, Han HS, Cho KH, Kim YB, Seo JW and Song CW Studies on the basic data of Ktc: C57BL/6 mice with age: body weight, organ weight, hematology, serum chemistry and urinalysis. Korea J Lab Anim Sci 10: 197-209, 1994.

14. Schnell MA, Hardy C, Hawley M, Propert KJ and Wilson JM: Effect of blood collection technique in mice on clinical pathology parameters. Hum Gene Ther 13: 155-161, 2002.

15. Vázquez-Vela ME, Torres $\mathrm{N}$ and Tovar AR: White adipose tissue as endocrine organ and its role in obesity. Arch Med Res 39: 715-728, 2008

16. Shin SS, Jung YS, Yoon KH, Choi S, Hong Y, Park D, Lee H, Seo BI, Lee HY and Yoon M: The Korean traditional medicine gyeongshingangjeehwan inhibits adipocyte hypertrophy and visceral adipose tissue accumulation by activating PPARalpha actions in rat white adipose tissues. J Ethnopharmacol 127: 47-54, 2010

17. Tchernof A: Visceral adipocytes and the metabolic syndrome. Nutr Rev 65: S24-S29, 2007.

18. Fasshauer M and Paschke R: Regulation of adipocytokines and insulin resistance. Diabetologia 46: 1594-1603, 2003.

19. Maffei M, Halaas J, Ravussin E, Pratley RE, Lee GH, Zhang Y, Fei H, Kim S, Lallone R, Ranganathan S, et al: Leptin levels in human and rodent: measurement of plasma leptin and ob RNA in obese and weight-reduced subjects. Nat Med 1: 1155-1161, 1995.

20. Rosen ED and Spiegelman BM: Adipocytes as regulators of energy balance and glucose homeostasis. Nature 444: 847-853, 2006.

21. Arita Y, Kihara S, Ouchi N, Takahashi M, Maeda K, Miyagawa J, Hotta K, Shimomura I, Nakamura T, Miyaoka K, Kuriyama H, Nishida M, Yamashita S, Okubo K, Matsubara K, Muraguchi M, Ohmoto Y, Funahashi T and Matsuzawa Y: Paradoxical decrease of an adipose-specific protein, adiponectin, in obesity. Biochem Biophys Res Commun 257: 79-83, 1999. 